\title{
SOME MORE UNIFORMLY CONVEX SPACES ${ }^{1}$
}

\author{
MAHLON M. DAY
}

Let $\left\{B_{i}, i=1,2, \ldots\right\}$ be a sequence of Banach spaces, and define $B=\mathcal{P}^{p}\left\{B_{i}\right\}$ to be the space of sequences $b=\left\{b_{i}\right\}$ with $b_{i} \in B_{i}$ and $\|b\|=\left(\left.\sum_{i}\left\|b_{i}\right\|\right|^{p}\right)^{1 / p}<\infty, 1<p<\infty$. It is known that $B$, normed in this way, is also a Banach space. Boas ${ }^{2}$ showed that if $B_{i}=l^{p}$ for all $i$ or if $B_{i}=L^{p}$ for all $i$ then $B$ is uniformly convex. Since then $I$ have shown ${ }^{3}$ that if $B_{i}=l^{p_{i}}$ or $L^{p_{i}}, 1<p_{i}<\infty$, and if the sequence $\left\{p_{i}\right\}$ is not bounded away from 1 and $\infty$ (that is, if there do not exist $1<m$ $\leqq M<\infty$ with $m \leqq p_{i} \leqq M$ for all $i$ ), then $B$ is not uniformly convex and cannot be renormed to an isomorphic uniformly convex space. One purpose of this note is to fill in the gap between these results by means of the following theorem.

Theorem 1. If $B_{i}=l^{p_{i}}$ or $L^{p_{i}}, 1<p_{i}<\infty$, and if the sequence $\left\{p_{i}\right\}$ is bounded away from 1 and $\infty$, then $B=\mathcal{P}^{p}\left\{B_{i}\right\}$ is uniformly convex.

Another question which is answered here is raised by Boas (loc. cit., Footnote 3). In Boas' notation $l^{p}\left(B_{0}\right)$ is the space $\mathcal{P}^{p}\left\{B_{i}\right\}$ with $B_{i}=B_{0}$ for all $i$. The space $L^{p}\left(B_{0}\right)$ is the space of all Bochner integrable functions $s^{4} f$ on, say, $[0,1]$ with values in $B_{0}$ and with $\|f\|=$ $\left[\int_{0}^{1}\|f(x)\|{ }^{p} d x\right]^{1 / p}<\infty$. Boas' conjecture is verified by the next theorem.

Theorem 2. $l^{p}\left(B_{0}\right)$ and $L^{p}\left(B_{0}\right)$ are uniformly convex if (and, obviously, only if) $B_{0}$ is.

Both of these results follow from the remaining theorem of this note. If all $B_{i}, i<\infty$, are uniformly convex there exists for each $\epsilon$, $0<\epsilon \leqq 2$, a positive number $\delta_{i}(\epsilon)$ such that $\left\|b_{i}\right\|=\left\|b_{i}^{\prime}\right\|=1$ and $\left\|b_{i}-b_{i}^{\prime}\right\|>\epsilon$ implies $\left\|b_{i}+b_{i}^{\prime}\right\|<2\left(1-\delta_{i}(\epsilon)\right)$. The sequence $\left\{B_{i}, i<\infty\right\}$ will be said to have a common modulus of convexity if there is one function $\delta(\epsilon)>0$ which can be used here in place of all $\delta_{i}(\epsilon)$. It is clear that if we define $\delta_{i}(\epsilon)=\frac{1}{2} \inf \left[2-\left\|b_{i}+b_{i}^{\prime}\right\|\right]$, where the infimum is taken over $b_{i}, b_{i}^{\prime}$ with $\left\|b_{i}\right\|=\left\|b_{i}^{\prime}\right\|=1$ and $\left\|b_{i}-b_{i}^{\prime}\right\|>\epsilon$, then such a $\delta(\epsilon)$ exists if and only if $\inf _{i} \delta_{i}(\epsilon)>0$ for every $\epsilon>0$. It is clear that $\delta(\epsilon)$ may be assumed to be a non-decreasing function of $\epsilon$.

${ }_{1}^{1}$ Presented to the Society, November 23, 1940.

2 R. P. Boas, Jr., Some uniformly convex spaces, this Bulletin, vol. 46 (1940), pp. 304-311.

${ }^{3}$ M. M. Day, Reflexive Banach spaces not isomorphic to uniformly convex spaces, this Bulletin, vol. 47 (1941), pp. 313-317.

${ }^{4} \mathrm{~S}$. Bochner, Integration von Funktionen, deren Werte die Elemente eines Vektorraumes sind, Fundamenta Mathematicae, vol. 20 (1933), pp. 262-276. 
Theorem 3. $B=\mathscr{P}^{p}\left\{B_{i}\right\}$ is uniformly convex if and only if the $B_{i}$ have a common modulus of convexity.

The space $B_{i}$ is equivalent to the closed linear subspace of those points of $B$ of the form $\left\{0,0, \ldots, 0, b_{i}, 0, \ldots\right\}$. If $B$ is uniformly convex, it follows that the function $\delta(\epsilon)$ existing in $B$ will be suitable as a common modulus for all the $B_{i}$.

The proof of the second part of Theorem 3 falls naturally into two cases. First we assume $\|b\|=\left\|b^{\prime}\right\|=1,\left\|b-b^{\prime}\right\|>\epsilon$ and $\left\|b_{i}\right\|=\left\|b_{i}^{\prime}\right\|$ for all $i$. For convenience let $\left\|b_{i}\right\|=\beta_{i}$ and $\left\|b_{i}-b_{i}^{\prime}\right\|=\gamma_{i}$. Then for any $i$, $\left\|b_{i}+b_{i}^{\prime}\right\|<2\left(1-\delta\left(\gamma_{i} / \beta_{i}\right)\right) \beta_{i}$ since $b_{i}$ and $b_{i}^{\prime}$ both lie on the sphere of radius $\beta_{i}$ about the origin in $B_{i}$. Hence

$$
\begin{aligned}
\left\|b+b^{\prime}\right\| & =\left(\sum_{i<\infty}\left\|b_{i}+b_{i}^{\prime}\right\| p\right)^{1 / p} \\
& \leqq 2\left\{\sum_{i}\left[\left(1-\delta\left(\gamma_{i} / \beta_{i}\right)\right) \beta_{i}\right]^{p}\right\}^{1 / p} .
\end{aligned}
$$

Also $\left\|b_{i}-b_{i}^{\prime}\right\| \leqq\left\|b_{i}\right\|+\left\|b_{i}^{\prime}\right\|$ so $\gamma_{i} \leqq 2 \beta_{i}$ for all $i$; let $E$ be the set of those $i$ 's such that $\gamma_{i} / \beta_{i}>\epsilon / 4$ and let $F$ be the complement of $E$. $\beta_{i} \geqq 4 \gamma_{i} / \epsilon$ if $i \in F$ so

$$
1=\left(\sum_{i<\infty} \beta_{i}^{p}\right)^{1 / p} \geqq\left(\sum_{i \in F} \beta_{i}^{p}\right)^{1 / p} \geqq(4 / \epsilon)\left(\sum_{i \in F} \gamma_{i}^{p}\right)^{1 / p}
$$

that is,

or

$$
\left(\sum_{i \in F} \gamma_{i}^{p}\right)^{1 / p} \leqq \epsilon / 4
$$

$$
\left(\sum_{i \in E} \gamma_{i}^{p}\right)^{1 / p}=\left(\sum_{i<\infty} \gamma_{i}^{p}-\sum_{i \in F} \gamma_{i}^{p}\right)^{1 / p} \geqq\left(\epsilon^{p}-(\epsilon / 4)^{p}\right)^{1 / p} \geqq 3 \epsilon / 4 .
$$

Hence

Using this and (1)

$$
\alpha=\left(\sum_{i \in E} \beta_{i}^{p}\right)^{1 / p} \geqq 3 \epsilon / 8 .
$$

$$
\begin{aligned}
\left\|b+b^{\prime}\right\| & \leqq 2\left[(1-\delta(\epsilon / 4))^{p} \sum_{i \in E} \beta_{i}^{p}+\sum_{i \in E} \beta_{i}^{p}\right]^{1 / p} \\
& \leqq 2\left[(1-\delta(\epsilon / 4))^{p} \alpha^{p}+1-\alpha^{p}\right]^{1 / p} \\
& \leqq 2\left\{1-\left[1-(1-\delta(\epsilon / 4))^{p}\right] \alpha^{p}\right\}^{1 / p} \\
& \leqq 2\left\{1-\left[1-(1-\delta(\epsilon / 4))^{p}\right](3 \epsilon / 8)^{p}\right\}^{1 / p}
\end{aligned}
$$


The coefficient of $\alpha^{p}$ is positive and depends only on $\epsilon$, so, if we set the right-hand side equal to $2\left(1-\delta_{0}(\epsilon)\right)$, then $\delta_{0}(\epsilon)>0$ if $\epsilon>0$, thus disposing of the first case.

In the general case we suppose only that $\|b\|=\left\|b^{\prime}\right\|=1$ and that $\left\|b+b^{\prime}\right\|>2\left(1-\delta_{1}(\alpha)\right)$, where $0<\alpha \leqq 2$ and $\delta_{1}(\alpha)$ is any modulus of convexity which can be used in the definition of uniform convexity in $l^{p}$. Then

$$
2\left(1-\delta_{1}(\alpha)\right) \leqq\left(\sum_{i}\left\|b_{i}+b_{i}^{\prime}\right\|^{p}\right)^{1 / p} \leqq\left(\sum_{i}\left(\left\|b_{i}\right\|+\left\|b_{i}^{\prime}\right\|\right)^{p}\right)^{1 / p} \leqq 2 .
$$

Since the sequences $\left\{\left\|b_{i}\right\|\right\}$ and $\left\{\left\|b_{i}^{\prime}\right\|\right\}$ are in $l^{p}$, we have

$$
\left(\sum_{i}\left(\left\|b_{i}\right\|-\left\|b_{i}^{\prime}\right\|\right)^{p}\right)^{1 / p} \leqq \alpha .
$$

Let $b^{\prime \prime}=\left\{b_{i}^{\prime \prime}\right\}$ be the point with $b_{i}^{\prime \prime}=b_{i}^{\prime}\left\|b_{i}\right\| /\left\|b_{i}^{\prime}\right\| ;$ then $\left\|b_{i}^{\prime \prime}\right\|$ $=\left\|b_{i}\right\|$ for each $i$ and $\left\|b^{\prime}-b^{\prime \prime}\right\|=\left(\sum_{i}\left(\left\|b_{i}^{\prime}\right\|-\left\|b_{i}\right\|\right)^{p}\right)^{1 / p}<\alpha$; hence, by Case I, $\left\|b-b^{\prime \prime}\right\| \leqq \epsilon / 2$ if $\left\|b+b^{\prime \prime}\right\| \geqq 2\left(1-\delta_{0}(\epsilon / 2)\right)$, but $\left\|b+b^{\prime \prime}\right\|$ $\geqq\left\|b+b^{\prime}\right\|-\left\|b^{\prime \prime}-b^{\prime}\right\|>2\left(1-\delta_{1}(\alpha)-\alpha / 2\right)$.

Take $\alpha$ so that $0<\alpha<\epsilon / 2$ and $\delta_{1}(\alpha)+\alpha / 2<\delta_{0}(\epsilon / 2)$; then $\left\|b+b^{\prime}\right\|$ $>2\left(1-\delta_{1}(\alpha)\right)$ implies $\left\|b+b^{\prime \prime}\right\|>2\left(1-\delta_{0}(\epsilon / 2)\right)$ which in turn implies $\left\|b-b^{\prime \prime}\right\|<\epsilon / 2$, leading finally to $\left\|b-b^{\prime}\right\| \leqq\left\|b-b^{\prime \prime}\right\|+\left\|b^{\prime}-b^{\prime \prime}\right\|<\epsilon / 2$ $+\epsilon / 2$. Let the $\delta_{1}(\alpha)$ defined by this process be $\delta_{2}(\epsilon)$; this is greater than zero and $\left\|b-b^{\prime}\right\|<\epsilon$ if $\|b\|=\left\|b^{\prime}\right\|=1$ and $\left\|b+b^{\prime}\right\|>2\left(1-\delta_{2}(\epsilon)\right)$. This proves Theorem 3 .

To show that Theorem 1 follows from this, use Clarkson's evaluations $^{5}$ of $\delta_{p}(\epsilon)$ in $l^{p}$ or $L^{p}: \delta_{p}(\epsilon)=1-\left[1-(\epsilon / 2)^{r}\right]^{1 / r}$ where $r=p$ if $p \geqq 2$ and $r=p /(p-1)$ if $1<p \leqq 2$. The sequence $\left\{p_{i}\right\}$ with $1<p_{i}<\infty$ is bounded away from 1 and $\infty$ if and only if the sequence $\left\{r_{i}\right\}$ associated with $\left\{p_{i}\right\}$ is bounded. A little elementary calculus will convince the reader that if $\epsilon$ is fixed $\delta_{p}(\epsilon)$ decreases as $r$ increases; hence if $r \geqq r_{i}$ for all $i, \delta_{r}(\epsilon) \leqq \delta_{p_{i}}(\epsilon)$ for all $i$ and the sequence of spaces $\lambda^{p_{i}}$ satisfies the hypothesis of Theorem 3 for $\lambda^{p_{i}}=l^{p_{i}}$ or $L^{p_{i}}$ provided only that $\left\{p_{i}\right\}$ is bounded away from 1 and $\infty$.

The first part of Theorem 2, that $l^{p}\left(B_{0}\right)$ is uniformly convex if $B_{0}$ is, is a trivial consequence of Theorem 3 . The proof for $L^{p}\left(B_{0}\right)$ involves an embedding argument similar to that used by Clarkson in analogous circumstances in $L^{p}$.

Let $H$ be the class of simple functions in $L^{p}\left(B_{0}\right)$; that is, $h \in H$ if

${ }^{5} \mathrm{~J}$. A. Clarkson, Uniformly convex spaces, Transactions of this Society, vol. 40 (1936), pp. 396-414. 
there exist measurable sets $E_{1}, \cdots, E_{n}$, disjoint, whose sum is the unit interval, and points $h_{1}, \cdots, h_{n} \in B_{0}$ such that $h=\sum_{i \leqq n} h_{i} \phi_{E_{i}}$ where $\phi_{E_{i}}$ is the characteristic function of the set $E_{i}$; that is, $\phi_{E_{i}}(t)=1$ if $t \in E_{i}$ and is otherwise $0 . H$ is dense in $L^{p}\left(B_{0}\right)$. If $E_{1}, \cdots, E_{n}$ are fixed, set up a correspondence between all functions in $H$ of the form $h=\sum_{i \leqq n} h_{i} \phi_{E_{i}}$ and all points $h$ in $l^{p}\left(B_{0}\right)$ with coordinates $b_{i}=0$ for $i>n$ as follows : $h$ corresponds to $h=\left\{b_{i}\right\}$ if $b_{i}=h_{i} \beta_{i}^{1 / p}$ for $i \leqq n$ where $\beta_{i}=m\left(E_{i}\right), \quad b_{i}=0$ otherwise. Then $\|h\|=\left(\sum_{i \leqq n} h_{i}^{p} \beta_{i}\right)^{1 / p}=\|h\|$ so the transformation is 1 -to-1 and norm preserving.

If $h, h^{\prime}$ are of the given form, if $\|h\|=\left\|h^{\prime}\right\|=1$ and $\left\|h-h^{\prime}\right\|>\epsilon$, then the same relations are true for the points $\boldsymbol{h}, \boldsymbol{h}^{\prime}$ in $l^{p}\left(B_{0}\right)$; so $\left\|h-h^{\prime}\right\|$ $=\left\|\boldsymbol{h}-\boldsymbol{h}^{\prime}\right\|<2(1-\delta(\boldsymbol{\epsilon}))$ where $\delta(\boldsymbol{\epsilon})$ is the modulus of convexity in $l^{p}\left(B_{0}\right)$. Now for any pair $h, h^{\prime}$ of functions in $H$ there is a partition of the unit interval into sets $E_{1}, \cdots, E_{n}$ such that $h, h^{\prime}$ are represented in the proper form using these sets. It follows that the inequality given holds for any $h, h^{\prime} \in H$ with $\|h\|=\left\|h^{\prime}\right\|=1$ and $\left\|h-h^{\prime}\right\|$ $>\epsilon$. Hence by continuity of the norm in $L^{p}\left(B_{0}\right)$ and by the density of $H$ in $L^{p}\left(B_{0}\right), L^{p}\left(B_{0}\right)$ is uniformly convex and the same modulus of convexity holds in it as in $l^{p}\left(B_{0}\right)$.

We may remark that the use of the interval $(0,1)$ and Lebesgue measure was obviously non-essential; any measure finite or infinite on any Borel field of measurable subsets of any set might be used with integration as might be expected. The essential property of the type of integration is that the simple functions in $L^{p}\left(B_{0}\right)$ shall be dense there.

It is also noteworthy that in the proof of the last part of Theorem 2 we gave the essential part of the proof of the following corollary.

Corollary 1. For fixed $p$ with $1<p<\infty$ and fixed uniformly convex $B_{0}$ the spaces $l^{p}\left(B_{0}\right)$ and $L^{p}\left(B_{0}\right)$ (no matter what measure is used in defining the space $L^{p}\left(B_{0}\right)$ ) have a common modulus of convexity, that of, for example, $l^{p}\left(B_{0}\right)$.

In fact, since the evaluation of $\delta_{2}(\epsilon)$ in Theorem 3 used only $\delta(\epsilon)$ in the spaces $B_{i}$ and $\delta_{1}(\epsilon)$ in $l^{p}$, it follows for $p$ in any interval $1<m \leqq p$ $\leqq M<\infty$ and for any $\left\{B_{i}\right\}$ or $B$ from a family of spaces $\{B\}$ with common modulus of convexity, that $\mathscr{P}^{p}\left\{B_{i}\right\}$ and hence $l^{p}(B)$ and $L^{p}(B)$ all have a common modulus of convexity.

\section{UNIVERSITY OF ILLINOIS}

